Electrooptic Characterization of Tunable Cylindrical Liquid Crystal Lenses

V. Urruchi , J. F. Algorri , J. M. Sánchez-Pena , N. Bennis , M.

A. Geday \& J. M. Otón 


\title{
Electrooptic Characterization of Tunable Cylindrical Liquid Crystal Lenses
}

\author{
V. URRUCHI, J. F. ALGORRI, J. M. SÁNCHEZ-PENA, \\ N. BENNIS, M. A. GEDAY, AND J. M. OTÓN
}

\begin{abstract}
In this work, one-dimensional arrays of cylindrical adaptive liquid crystal lenses were manufactured and characterized; and test devices were filled with nematic liquid crystal. Comb interdigitated electrodes were designed as a mask pattern for the control electrode on the top glass substrates. A radial graded refractive index along each microsized lens was achieved by fabricating a layer of high resistance sheet deposited as a control electrode. These tunable lenses were switched by applying amplitude and frequency optimized waveforms on the control electrode. Phase profiles generated by the radial electric field distribution on each lens were measured by a convectional interferometric technique.
\end{abstract}

Keywords Liquid crystal lens; optimized waveforms; phase measurement; graded index

\section{Introduction}

Since the first tunable lenses based on liquid crystal (LC) technology were proposed more than 30 years ago, research on specific fabrication techniques for those devices has been developed in depth. The electrical control of the focal length becomes a key requirement for all approaches compared to conventional lenses with fixed focal length. In this sense, high birefringence LC materials may contribute to the design of wide range operation devices. On the other hand, by reducing the size of the lenses to a micrometric scale with array structures, one- or two-dimensional, the range of applications can be extended further. Specifically, dynamic lenses play an important role in optical interconnection, adaptive electrooptic components, beam steering, vision corrective elements in spectacles or image integration components in autostereoscopic displays $[1,2]$. Moreover, the relevance of LC lenses arise from their small size, light weight, low control voltage, low power consumption and transmissive/reflective operation modes. Currently, the main drawbacks are slow response time (ms) and electrooptic behavior dependent on polarization. 
Several approaches to LC lens design have been reported, such us using polymer stabilization LC techniques [3, 4], hole patterned electrode schemes [5, 6], surface relief profiles [7] or modal control addressing. The first researches on modal control were reported by A.F. Naumov et al. [8]. This technique consists of generating a radial graded refractive index across each lens of the array by using a layer of high resistance sheet $(\mathrm{M} \Omega / \mathrm{sq}$ ), deposited onto the pattern electrode, as a control electrode $[9,10]$. In this work, we have proposed a special mask electrode pattern (comb interdigitated) for a tunable cylindrical lenticular lens array with modal control. This scheme has allowed us to compare phase retardation of two lenticular approaches via the same manufactured device. Both lenticular arrays share identical manufacturing protocol, except that the first approach owns double lenticular pitch than the second one.

\section{Cylindrical Lens Design}

The key of the modal control is to achieve a suitable design of a high resistivity control electrode and, especially, to manage the selection of appropriate materials for device manufacturing. The typical layer structure of a modal device consists of: a low resistivity electrode (working like a counter electrode)/a LC layer/a high resistivity electrode + a mask pattern (working like a control electrode). A high resistivity layer can be electrically modeled as a transmission line that connected to a resistor-capacitor equivalent circuit for a LC layer, performs a uniform electric field gradient from the center to the edges of a lens. Such a proposed simple model could be described as a chain of successive voltage dividers from the junction of the impedance properties of both circuits. But in addition, reactance of equivalent capacitor is responsible for the frequency response of tunable device.

Some of the usual drawbacks of the materials for the control electrode are related to stability and repeatability of layers. So, some conditions must be met for the material to be used. As reported in the literature, sheet resistance must be in the range $100 \mathrm{k} \Omega / \mathrm{sq}-$ $1 \mathrm{M} \Omega / \mathrm{sq}$ for implementing a right voltage divider with LC equivalent circuit. Candidate material should be chemically compatible with surrounding materials. Finally, manufacturing protocols for constructing tunable devices should be repeatable for the selected materials.

\section{Control Electrode Proposal}

One of the most common materials reported for high resistivity is PEDOT [9]. In this work, we have introduced a new kind of material for designing the control electrode. The proposal consists of a film of a metallic material thin enough to behave like a high resistance electrode. Resistivity of metallic materials have been reported [11] to experience a nonlinear growing according to the decreasing of the material thickness layer, specifically if a nanometric range for the layer thickness is considered. This feature, the electric resistivity is known as an intrinsic material property that shows us its capacity of electric response. However, near to those thicknesses the resistivity increases exponentially, so we have taken advantage of this behavior for designing some high resistivity electrodes. Nickel has been the selected material: it is low cost and can be deposited by an evaporation process.

Some glass substrates have been manufactured with the appropriate control electrode. Previous characterization of sheet resistance of those layers has been studied before assembling the final lenticular lenses. Conventional Four-Point Probe Method has been used for 


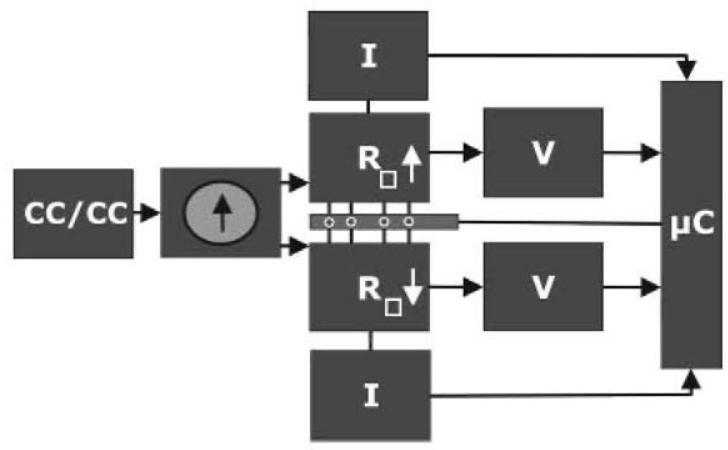

Figure 1. Diagram of electrical scheme implemented for measuring sheet resistances of thin layers.

measuring sheet resistances. This property can be measured by the following equation on thin layers,

$$
\mathrm{R}_{\mathrm{sq}}=\frac{\pi}{\ln 2} \cdot \frac{\mathrm{V}}{\mathrm{I}}
$$

where $\mathrm{V}$ and I are the voltage and current, respectively, applied to the measurement system. A specific circuit has been implemented for measuring properly the values of sheet resistance. The block diagram of Fig. 1 summarizes the function of the electrical design. This circuit is based on a current source (circle symbol with an arrow inside) that will go through the high resistivity layer by a measuring head connected to a four-point probe (symbol with four circles). Circuit design has been split into two parts, each of them specially optimized for high or low sheet resistance measurements, respectively. The top part of the circuit is dedicated to measuring high sheet resistance $(\mathrm{M} \Omega / \mathrm{sq})$; in contrast, the bottom part has been implemented for measuring low sheet resistance $(\Omega / \mathrm{sq})$. This scheme allows the system to reduce measurement errors. Also, circuit requires a high DC power supply to operate (near 40 volts), so a boost converter $(\mathrm{CC} / \mathrm{CC})$ has been implemented. Finally, a microcontroller $(\mu \mathrm{C})$ records the ratio voltage/current and reports about the experimental sheet resistance.

Sheet resistance $(\Omega / \mathrm{sq})$ has been measured for several control electrodes with different high resistivity layers. Moreover, if the sample thickness is known, electrical resistivity can be estimated as well. Figure 2 shows that layers with a thickness under 70 angstroms experience a sharp growing of its sheet resistance. Sheet resistances obtained are within the range proposed in the literature for designing high resistivity control electrodes.

Finally, a crucial characteristic of the lenses is the light transmittance through them. In this sense, proposal of metal electrodes is not an inconvenience. Despite of metallic films are normally opaque, at nanometric scale, as sheet resistance increases, transmittance also increases. In order to characterize the transmittance, two luminance measurements were done with a luminance meter. The first measurement was the luminance of a homogeneous white lamp, giving rise $60 \mathrm{~cd} / \mathrm{m}^{2}$. The second one was carried out with the lenticular array onto the lamp (used as a light source), obtaining $48 \mathrm{~cd} / \mathrm{m}^{2}$. Ratio between those values was $80 \%$, assuming nickel as alternative candidate for high resistivity electrodes in LC lenses. 


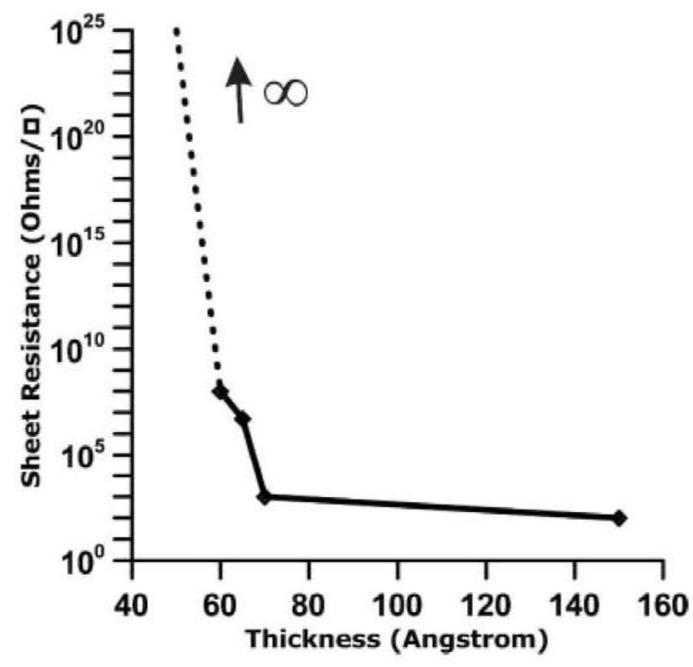

Figure 2. Thickness layer dependence of sheet resistance $(\Omega / s q)$ of thin nickel layers.

\section{Chrome Mask Design}

Chrome mask patterns consisted of one-dimensional arrays of cylindrical lenses designed with two comb interdigitated electrodes. This versatile design allowed the simultaneous arrangement of two arrays sharing the same fabrication protocol. The former approach has double lens pitch by applying the electric field only through one comb electrode; however, for the second, the two interdigitated electrodes are used for the device driving (Fig. 3).

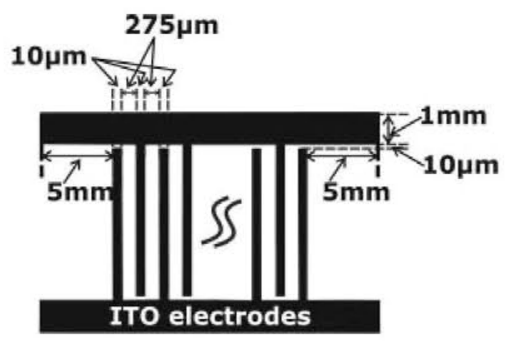

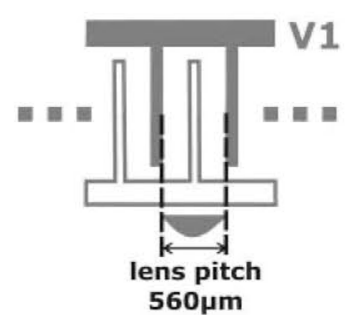

(a)

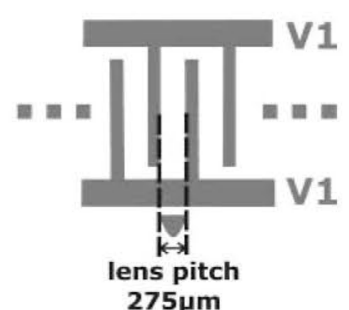

(b)

Figure 3. Chrome masks design based on two comb interdigitated electrodes as a control electrode. a) First addressing approach: A double lens pitch by applying electric field only to one comb electrode. b) Second addressing approach: A half lens pitch by applying electric field to two comb electrodes. 
The initial criteria of the mask design was a focal length $=3 \mathrm{~mm}$ for focusing light into a near slit array. Thus, considering each lens of the array mimics the effect of a gradient index lens (GRIN), the focal length of each ( $\mathrm{f}_{\mathrm{GRIN}}$ ) can be derived by equation

$$
\mathrm{f}_{\mathrm{GRIN}}=\frac{\mathrm{r}^{2}}{2 \cdot \mathrm{d} \cdot\left(\mathrm{n}_{\max }-\mathrm{n}(\mathrm{r})\right)}
$$

where $\mathrm{r}$ is the radius of a lens (half pitch lens), $\mathrm{d}=50 \mu \mathrm{m}$ is the thickness of the LC cell and $n_{\max }-n(r)$ is the difference between the maximum refractive index, $n_{\max }$ (at the optical axis of each lens) and the refractive index at the position $r$ (that is, at the edge of the lens). Additionally, since the homogeneous alignment of the lenses is combined to the electric field gradient through the lens surface, $\mathrm{n}_{\max }$ identifies to the extraordinary index, ne $=1.7779$, and $n(r)$ to the ordinary index, no $=1.5113$, for the nematic liquid crystal MDA-98-1602 $(\Delta \mathrm{n}=0.2666)$ from Merck. Hence, theoretical lens radius for the first approach ( $\mathrm{f}_{\mathrm{GRIN}}=3 \mathrm{~mm}$ ), can be obtained according to the previous equation as $\mathrm{r}=$ $285 \mu \mathrm{m}$. On the other hand for the second approach, a half lenticular pitch forces the focal length to decrease until $\mathrm{f}_{\mathrm{GRIN}}=0.8 \mathrm{~mm}$, so lens radius, $\mathrm{r}=142.5 \mu \mathrm{m}$.

Lens radius sizes, for each approach, are the minimum requirements for designing the mask proposal (Fig. 3) knowing the size of electrode columns and gaps between electrodes.

\section{Experimental Set-Up}

Phase retardation, $\Phi$, has been measured by a conventional interference method. The experimental setup is shown in Fig. 4.

Interference fringes were captured placing test samples between two crossed polarizers with the rubbing direction of the lenticular lenses at $45^{\circ}$ from the linear polarization at the input. A variable frequency and amplitude square signal was applied to the control electrode of the LC lenticular lenses. A polarized He-Ne laser beam $(\lambda=632.8 \mathrm{~nm})$ was used as a light source. The first polarizer splits incident light wave into two orthogonal components, the extraordinary and ordinary waves. When light passes through the LC device, waves

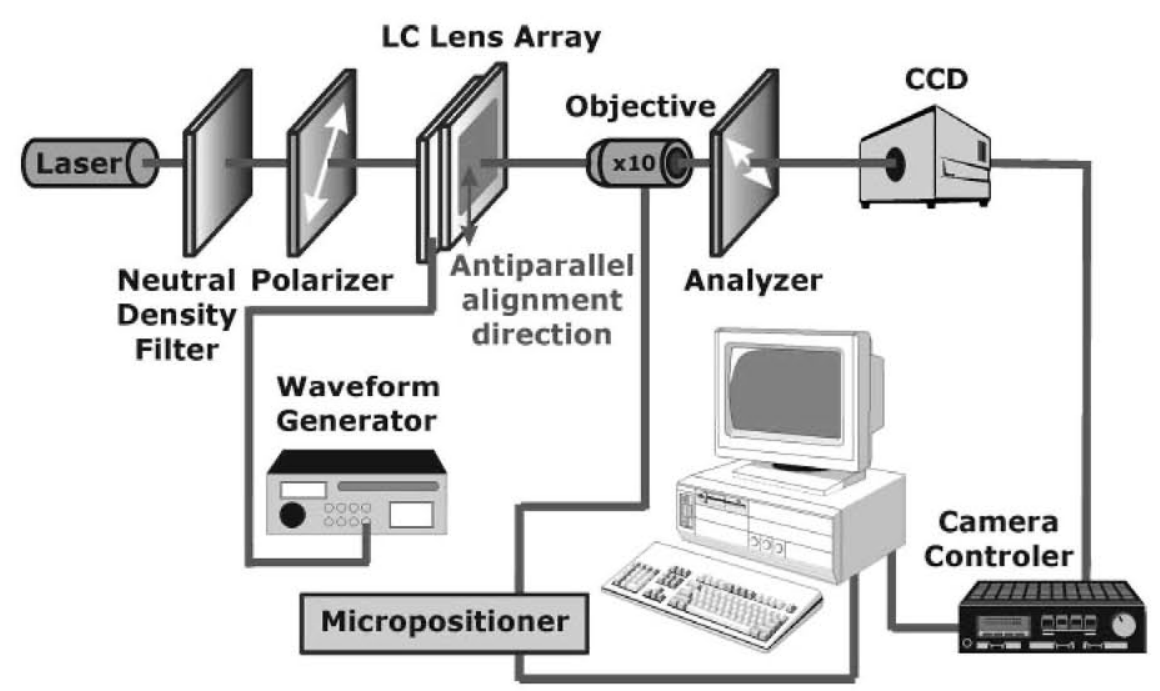

Figure 4. Experimental set-up for electrooptic characterization of phase retardation. 
experience spatially uniform phase retardation caused by the birefringence of LC. As a gradient voltage has been designed to be generated inside the LC device, a gradient birefringence is also generated. At this point, second polarizer recombines the two waves and causes them to interfere. Wave result was an interference pattern composed of darks and clear zones corresponding to constructive and destructive interferences. This means that two neighboring interference fringes have a phase difference of $2 \pi$. Unlike other lens, where lens aperture is visible to the naked eye, these lenses have a micrometer pitch length. A $\times 10$ objective properly focused was placed in the path of the beam to see correctly the interference pattern handling this task with a micro-positioning. Finally, interference fringes were recorded by a CCD camera as applying various voltages and frequencies square signals. Phase profiles were obtained from interference patterns developing an image recognition program that processes images and plots phase retardation versus horizontal position across the LC lens.

\section{Charectarization of Phase Retardation}

Results of characterization for first addressing approach (for applied signals only to one of the comb electrodes) have allowed a thorough study of the manufacturing protocol together with the amplitude and frequency dependence on the phase behavior of the lenticular device. Figure 5 includes interference fringes for some representative values of frequency and voltages (root mean square) of addressing waveform, so width of each image matches with a lens diameter. It is shown that, for a fixed frequency, as voltage increases, phase differences spread out over the lens surface. However, lens diameter designed for this approach turned out too long $(570 \mu \mathrm{m})$, forcing high driving voltages and given rise to undesirable shrinking effect. It means that voltage distribution drops bellow threshold before reaching the center of the lens, thus suggesting a too large sheet resistance of the control electrode for these approach.

Shrinking effect resulted in a significant decreasing aperture diameter of lenses, probably due to a saturation of the tilt angle of molecules near the edges. This result also restricts
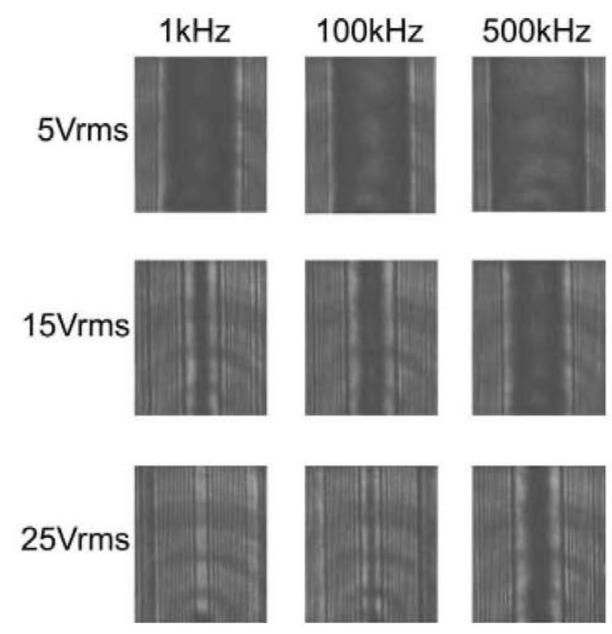

Figure 5. Frequency and voltage (rms) dependence of interference patterns of cylindrical LC lens when only one of the comb electrodes is used as a control electrode. 

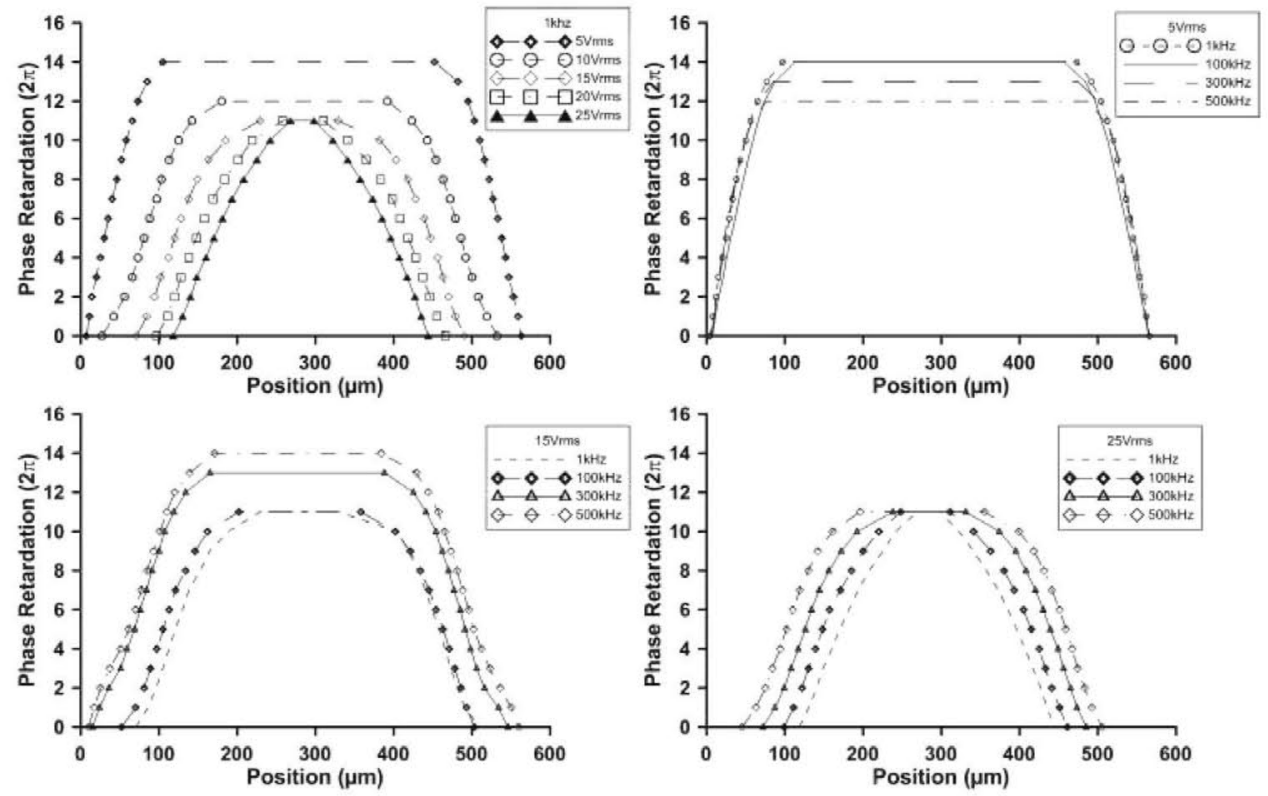

Figure 6. Frequency and voltage dependence of phase retardation of cylindrical LC lens when only one comb electrode is used as a control electrode.

the maximum effective phase retardation $(11 \pi$ ), as shown in Fig. 6 (upper left graph, $1 \mathrm{kHz}$ frequency AC square signal).

On the other hand, another noticeable effect, for a fixed voltage, is the growing of the flat-like phase in the center of the lens, that is, without any change in retardation phase, as frequency increases. Figure 6 shows some examples of this behavior for $5 \mathrm{~V}_{\mathrm{rms}}, 15 \mathrm{~V}_{\mathrm{rms}}$ and $25 \mathrm{~V}_{\mathrm{rms}}$ and several frequencies. Such an effect is related to the frequency dependence of the electrical response of manufactured devices. LC impedance, from the control electrode to the counter electrode, experiences a drop as frequency increases. A simple model of the equivalent electric circuit of nematic LC crystals explains that LC capacitance tends to shorting at high frequencies, so dropping the electric field at the center of the cell.

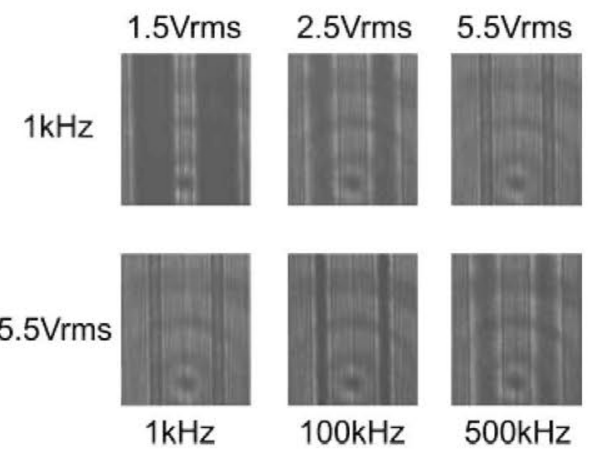

Figure 7. Frequency and voltage (rms) dependence of interference patterns of cylindrical LC lens when two comb electrodes are used as a control electrodes. 

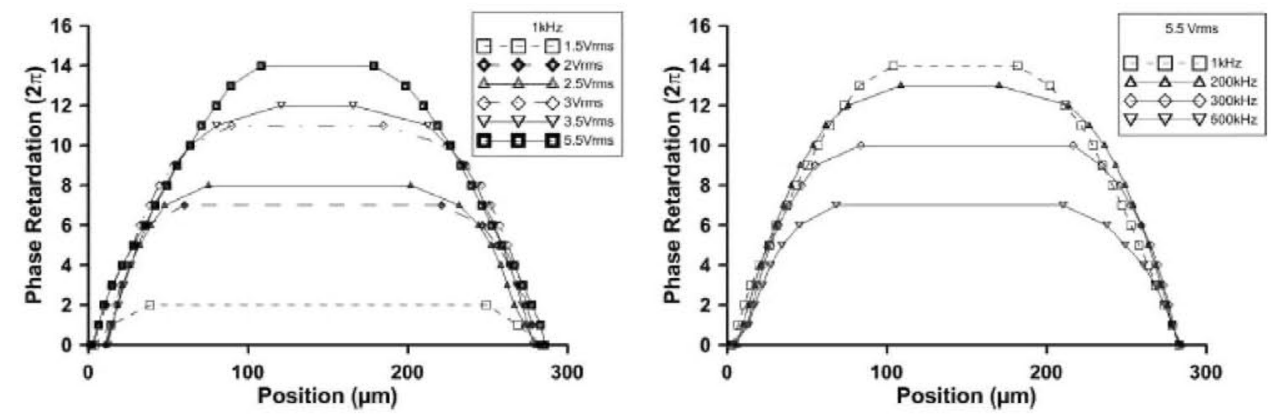

Figure 8. Frequency and voltage dependence of phase retardation of cylindrical LC lens when two comb electrodes are used as a control electrode.

Performance of first addressing approach (leading one comb electrodes) can be improved through the second addressing approach. By leading two comb electrodes allows us to design a lenticular array with optimum features. In this case, lens diameter is the half of the previous one $(285 \mu \mathrm{m})$, so that it becomes a suitable arrangement of lens size and sheet resistance of the control electrode. Unlike first approach, AC square driving applied to the current device configuration gives rise homogeneous phase responses within a full range of voltages and frequencies. Figure 7 shows interference fringes recorded for some representative values of frequency and voltages of AC signals. Width of each image matches with two lens diameter (quantitatively it is the same width than in Fig. 5).

As expected, for a fixed frequency, as voltage increases phase retardation profiles increase (focal length decreases). Maximum phase retardation is reached at the lens center (experimentally $14 \pi$ ) and minimum at the edges (Fig. 8). Lower voltages than those of the previous approach were used, avoiding shrinking effect appears. Minimum focusing distance whereby the maximum phase retardation is achieved has been obtained with only $5 \mathrm{~V}_{\text {rms }}$. Again, for a fixed voltage, similar frequency dependence of interference patterns happens. To finish, by tuning voltage and frequency parameters, a good choice for the optimal operating point in the second approach may be consider the pair $\left(5.5 \mathrm{~V}_{\mathrm{rms}}, 1 \mathrm{kHz}\right)$.

\section{Conclusions}

A cylindrical lens array has been reported for the first time using a new manufacturing protocol based on a metal layer for a lens with modal driving. A metal layer acts as a high resistivity electrode and generates a distributed resistance across the surface of the lens pitch from the centre to the edge. Metallic films thicknesses are a key parameter for designing a suitable voltage distribution through the inner surface of the control electrode. Amplitude and frequency of square AC signals have been optimized. Higher voltages give higher phase retardation and focusing powers. Too wide lens pitches (for a specific metal sheet resistance) force higher driving voltages. Too high voltages give rise to undesirable shrinking effect.

\section{Acknowledgments}

This work was supported in part by the Ministerio de Ciencia e Innovación of Spain (grant no.TEC2009-13991-C02-01) and Comunidad de Madrid (grant no. S2009/ESP-1781). 


\section{References}

[1] Hong, H.-K., Jung, S.-M., Lee, B.-J., Im, H-J., \& Shin, H.-H. (2008). Proceedings of SID International Symposium Digest of Technical Papers 08, 348-351.

[2] Huang, Y.-P., Chen, C.-W., Shen, T.-C., \& Huang, J.-F. (2010). Journal of 3D Research, 39-42.

[3] Presnyakov, V. V., Asatryan, K. E., Galstian, T. V., \& Tork, A. (2002). Opt. Express, 10(17), $865-870$.

[4] Kinoshita, M., Ikeda, T., Kobayashi, T., Takano, K., \& Nam, Y. (2010). SPIE Newsroom, DOI: 10.1117/2.1201009.003087.

[5] Ye, M., Wang, B., Kawamura, M., \& Sato, S. (2007). Jpn. J. Appl. Phys., 46(10A), 6776-6777.

[6] Ye, M., Wang, B., \& Sato, S. (2008). Opt. Express, 16(6), 4302-4308.

[7] Choi, Y., Kim, Y.-T., Lee, S.-D., \& Kim, J.-H. (2005). Mol. Cryst. Liq. Cryst., 433, 191-197.

[8] Vdovin, G. V., Guralnik, I. R., Zayakin, O. A., Klimov, N. A., Kotova, S. P., Loktev, M. Yu., Naumov, A. F., Patlan, V. V., \& Samagin, S. A. (2008). ISSN 1062-8738, Bulletin of the Russian Academy of Sciences, Physics, 72(1), 71-77.

[9] Fraval, N., Joffre, P., Formont, S., \& Chazelas, J. (2009). Appl. Opt., 48(28), 5301-5306.

[10] Fraval, N., \& de Bougrenet de la Tocnaye, J. L. (2010). Appl. Opt., 49(15), 2778-2783.

[11] Aus, M. J., Szpunar, B., Erb, U., \& El-Sherik, A. M. (1993). J. Appl. Phys., 75(7), 3632-3634. 